\title{
A new analysis of the combined data from both KASCADE and KASCADE-Grande
}

S. Schoo ${ }^{1}$, D. Kang ${ }^{1 *}$, W.D. Apel ${ }^{1}$, J.C. Arteaga-Velázquez ${ }^{2}$, K. Bekk $^{1}$, M. Bertaina ${ }^{3}$, J. Blümer ${ }^{1,4}$, H. Bozdog ${ }^{1}$, I.M. Brancus ${ }^{5}$, E. Cantoni ${ }^{3,6}$, A. Chiavassa ${ }^{3}$, F. Cossavella ${ }^{4}$, K. Daumiller ${ }^{1}$, V. de Souza ${ }^{7}$, F. Di Pierro ${ }^{3}$, P. Doll ${ }^{1}$, R. Engel ${ }^{1}$, D. Fuhrmann ${ }^{8}$, A. Gherghel-Lascu ${ }^{5}$, H.J. Gils ${ }^{1}$, R. Glasstetter ${ }^{8}$, C. Grupen ${ }^{9}$, A. Haungs ${ }^{1}$, D. Heck ${ }^{1}$, J.R. Hörandel ${ }^{10}$, D. Huber ${ }^{4}$, T. Huege ${ }^{1}$, K.-H. Kampert ${ }^{8}$, H.O. Klages ${ }^{1}$, K. Link ${ }^{4}$, P. Łuczak ${ }^{11}$, H.J. Mathes ${ }^{1}$, H.J. Mayer ${ }^{1}$, J. Milke ${ }^{1}$, B. Mitrica ${ }^{5}$, C. Morello ${ }^{6}$, J. Oehlschläger ${ }^{1}$, S. Ostapchenko ${ }^{12}$, N. Palmieri ${ }^{4}$, T. Pierog ${ }^{1}$, H. Rebel ${ }^{1}$, M. Roth ${ }^{1}$, H. Schieler ${ }^{1}$, F.G. Schröder ${ }^{1}$, O. Sima ${ }^{13}$, G. Toma ${ }^{5}$, G.C. Trinchero ${ }^{6}$, H. Ulrich ${ }^{1}$, A. Weindl ${ }^{1}$, J. Wochele ${ }^{1}$, J. Zabierowski ${ }^{11}$ - KASCADE-Grande Collaboration

${ }^{1}$ Institut für Kernphysik, KIT - Karlsruhe Institute of Technology, Germany

${ }^{2}$ Universidad Michoacana, Inst. Física y Matemáticas, Morelia, Mexico

${ }^{3}$ Dipartimento di Fisica, Università degli Studi di Torino, Italy

${ }^{4}$ Institut für Experimentelle Kernphysik, KIT - Karlsruhe Institute of Technology, Germany

${ }^{5}$ Horia Hulubei National Institute of Physics and Nuclear Engineering, Bucharest, Romania

${ }^{6}$ Osservatorio Astrofisico di Torino, INAF Torino, Italy

${ }^{7}$ Universidade São Paulo, Instituto de Física de São Carlos, Brasil

${ }^{8}$ Fachbereich Physik, Universität Wuppertal, Germany

${ }^{9}$ Department of Physics, Siegen University, Germany

${ }^{10}$ Dept. of Astrophysics, Radboud University Nijmegen, The Netherlands

${ }^{11}$ National Centre for Nuclear Research, Department of Astrophysics, Lodz, Poland

${ }^{12}$ Frankfurt Institute for Advanced Studies (FIAS), Frankfurt am Main, Germany

${ }^{13}$ Department of Physics, University of Bucharest, Bucharest, Romania E-mail: donghwa.kang@kit.edu

KASCADE and KASCADE-Grande were detector arrays for the measurement of extensive air showers generated by high-energy cosmic rays in the PeV to EeV energy range. KASCADEGrande has been operated for more than two decades and completed the data taking at the end of 2013. Now, a new analysis of the combined data from the arrays of KASCADE and KASCADEGrande was performed, increasing both the effective area and the accuracy of the shower reconstruction. In addition, new versions of post-LHC hadronic interaction models are used to interpret the combined data. First results from the new combined analysis confirming earlier observations of spectral features, but also unveiling still existing inconsistencies of the models, will be discussed in this contribution.

35th International Cosmic Ray Conference

10-20 July, 2017

Bexco, Busan, Korea

* Speaker. 


\section{Introduction}

The goals of investigations of high-energy cosmic rays are to determine the mass composition, the energy spectrum, and the arrival direction of cosmic rays. These studies are important to understand the origin of cosmic rays, as well as their propagation and acceleration. In particular, measurements in the energy range of $\mathrm{PeV}$ to EeV covered by KASCADE and KASCADE-Grande give a clue to identify the transition region of galactic and extragalactic cosmic rays.

Extensive air shower arrays of KASCADE and KASCADE-Grande completed the data acquisition at the end of 2013 and the detector components are fully dismantled. Detailed analysis of more than 20 years measured data presents fruitful results: The all-particle energy spectrum reconstructed using the KASCADE data shows a knee-like structure due to a steepening of spectra of light elements [1]. The all-particle energy spectrum of KASCADE-Grande [2] shows some structures, which cannot described by a single power law: a concave behavior just above $10^{16} \mathrm{eV}$ and a small break at around $10^{17} \mathrm{eV}$, where a knee-like feature would be expected as the knee of the heavy primaries, mainly iron. In the reconstructed energy spectra for individual mass groups, the knee-like feature in the heavy primary spectrum is observed much more significantly at around $80 \mathrm{PeV}$ [3]. Further, an ankle-like structure is observed at an energy of $100 \mathrm{PeV}$ in the energy spectrum of light primary cosmic rays [4].

However, the analysis of the measured KASCADE and KASCADE-Grande data was performed independently until now. Thus the motivation for the combined analysis is the reconstruction of the energy spectra for all-particle and for individual mass groups by means of a larger fiducial area and a higher accuracy of the shower reconstruction in order to confirm earlier observations of spectral features. In addition, the most recent hadronic interaction model of EPOS-LHC is used to interpret the combined data.

\section{KASCADE and KASCADE-Grande}

KASCADE [5] was an extensive air shower array located at Karlsruhe Institute of Technology, Karlsruhe, Germany $\left(49.1^{\circ}\right.$ north, $8.4^{\circ}$ east, $110 \mathrm{~m}$ above sea level). The KASCADE-Grande [6] experiment was the extension of the original KASCADE array designed to measure primary cosmic rays up to $1 \mathrm{EeV}$.

The KASCADE array consisted of 252 scintillation detector stations with non-shielded and shielded detectors located on a grid of $200 \times 200 \mathrm{~m}^{2}$ for the measurement of the electromagnetic and muonic shower $\left(E_{\mu}>230 \mathrm{MeV}\right)$ components, independently. It measured primary cosmic rays in the energy range of about 0.5 to $100 \mathrm{PeV}$. The Grande array was covering an area of $700 \times 700$ $\mathrm{m}^{2}$ measured primary cosmic rays in the energy range of $10 \mathrm{PeV}$ to $1 \mathrm{EeV}$. It comprised 37 scintillation detector stations located on a hexagonal grid with an average spacing of $137 \mathrm{~m}$ for the measurements of the charged shower component. Each of the detector stations was equipped with plastic scintillators covering a total area of $10 \mathrm{~m}^{2}$ per station.

While the Grande detectors are sensitive to all charged particles, the KASCADE detectors measure separately the electromagnetic and muonic components due to the shielding above the muon counters. Therefore, we reconstruct the shower core position, the arrival direction, and the 


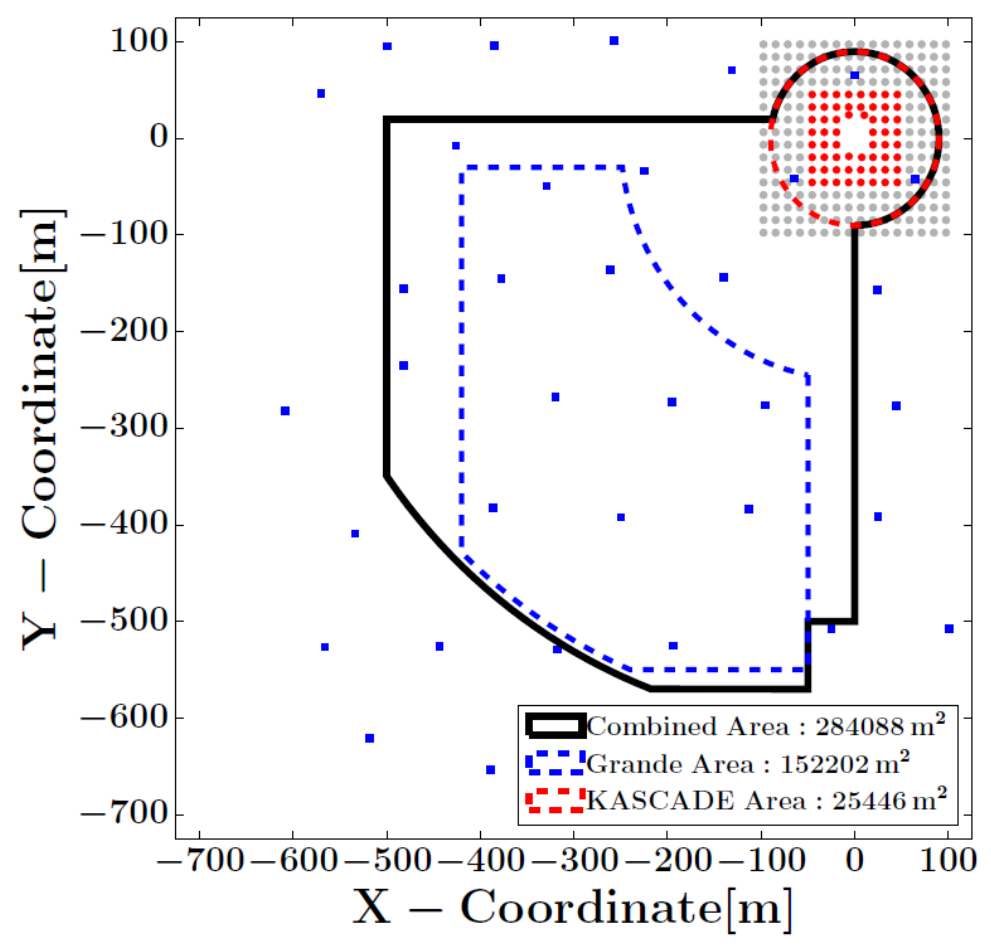

Figure 1: Schematic view of the KASCADE and KASCADE-Grande arrays. The gray area is covered by $622 \mathrm{~m}^{2}$ of muon detectors. The KASCADE central detector and muon tunnel are not shown, as not used in this analysis. The solid line shows the fiducial area for the combined analysis.

total number of charged particles in the shower from Grande array data, whereas the total number of muons is extracted from the data of the KASCADE muon detectors.

\section{Combined analysis of KASCADE and KASCADE-Grande data}

The crucial point for the combined analysis [7] is the shower reconstruction, which is done from the events measured by both KASCADE and KASCADE-Grande arrays. We perform the combined analysis of both array data with an extension of the fiducial areas (solid line) shown in Fig. 1. Events located in the Grande array gain 252 additional density measurements and events located in the KASCADE array gain 37 additional measurements. Therefore, the additional stations at larger distance allow us to reach higher energies for KASCADE located events and higher accuracy for Grande located events. It also allows a consistent reconstruction of the shower parameters over a wide energy range from $10^{15}$ up to $10^{18} \mathrm{eV}$. Above all, the main goal for combining the analysis is to obtain the all-particle and mass group spectra by one consistent reconstruction procedure.

\subsection{Shower reconstruction}

The shower reconstruction of the combined data follows the standard procedure of KASCADE and KASCADE-Grande [8]. We measure the energy deposited in the scintillation detectors and 


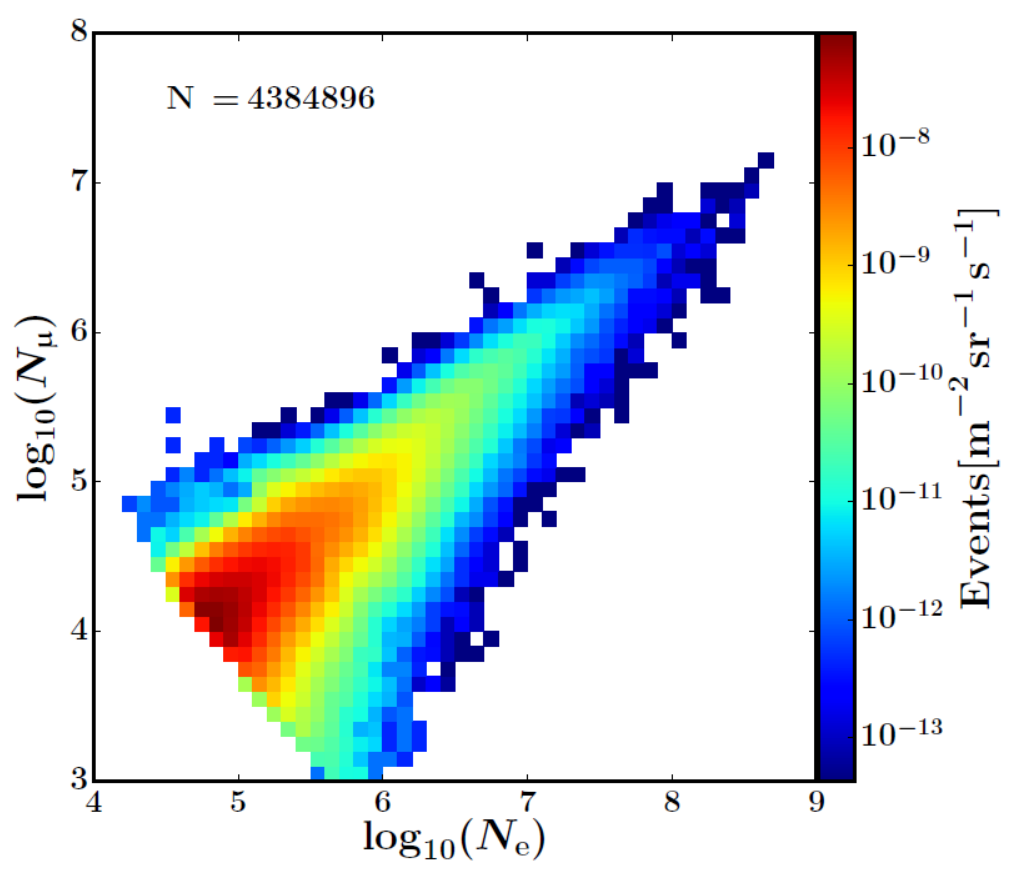

Figure 2: The 2-dimensional shower size distribution for the combined analysis obtained by a combined shower reconstruction of KASCADE and KASCADE-Grande data.

transform them into particle numbers. This is done by applying a lateral energy correction function, which describes the energy dependence of the stopping power and the energy release originated from photon conversion in the detector. Such a conversion is performed by means of simulations based on the CORSIKA code [9] and is nearly independent of the hadronic interaction model in use. The number of electrons $\left(N_{e}\right)$ is obtained by fitting a modified NKG function [10] to the lateral densities, while the muon number $\left(N_{\mu}\right)$ is based on the Lagutin function [11]. The reconstruction of the muon number is done by 192 muon detectors of the KASCADE arrays.

Due to the combination of two detector systems we reach a more accurate reconstruction of $N_{e}$ and $N_{\mu}$ than by the standalone analyses alone. The accuracy of the electron number at $10^{5}$ electrons improves about $6 \%$ for the array alone to about $4.5 \%$ for the combined reconstruction. The accuracy becomes better than 3\% at higher energies. The improvement on the muon reconstruction by means of combining both detector arrays is rather small at the threshold of the full efficiency. The reconstruction accuracy for the muon numbers are improved from about $25 \%$ to about $17 \%$.

The combined reconstruction leads to an improved accuracy over the whole range [12], i.e. it results in more accurately estimated observables.

\subsection{Energy estimation}

Figure 2 shows the 2-dimensional shower size spectrum obtained by the combined shower reconstruction from the data of both arrays. This distribution from a larger fiducial area is reconstructed with higher accuracy and covers more than three orders of magnitude in the primary 


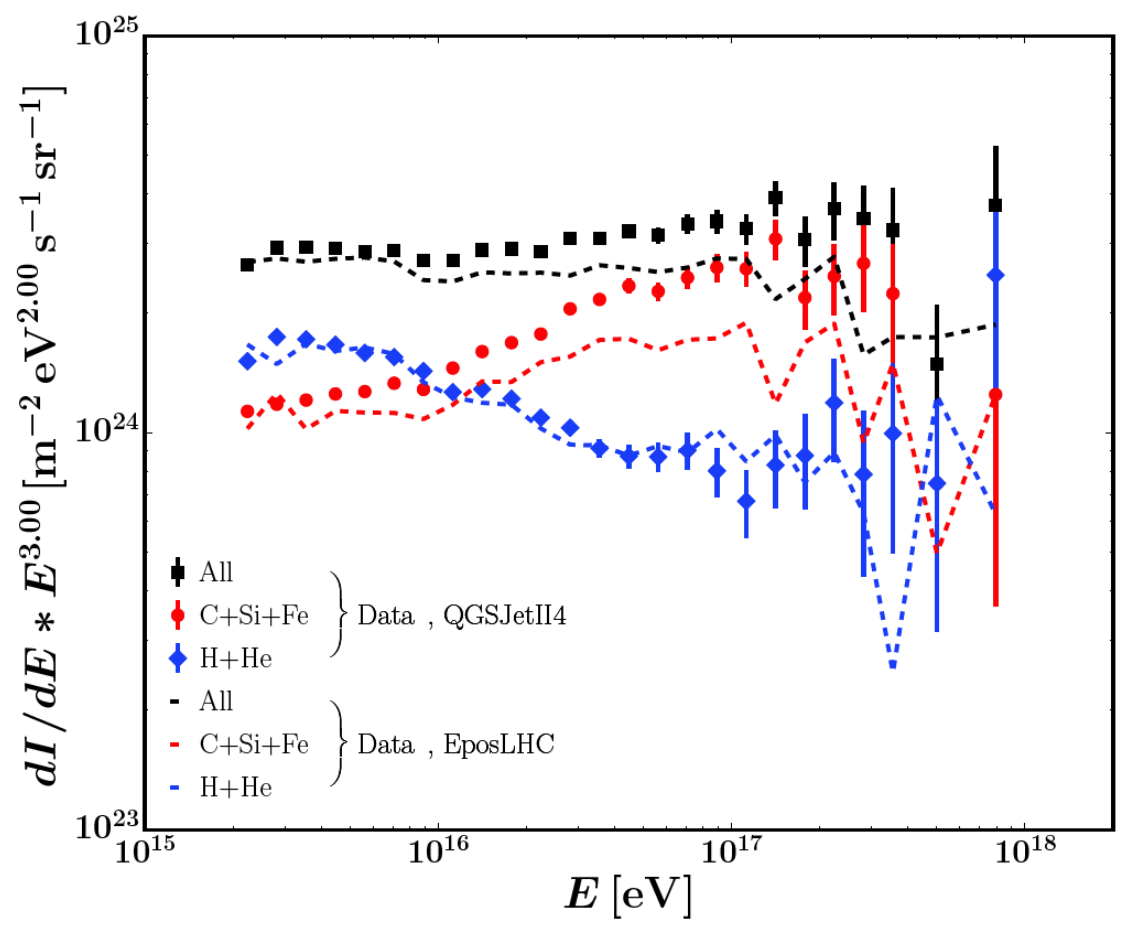

Figure 3: Reconstructed energy spectra from the combined analysis for the heavy and light components using energy calibrations based on EPOS-LHC (dashed lines) and QGSJet-II-04 (marks) interaction models.

energy. For this analysis we use 4384896 reconstructed events with a zenith angle of $0^{\circ}$ to $30^{\circ}$. From this event sample based on the number of electrons and muons at the observation level we perform the further analysis.

For the combined analysis, the $k$-parameter method [2] is applied to the combined reconstructed 2-dimensional shower size spectrum to obtain the all-particle energy spectrum, as well as the spectra of different mass groups. This $k$-parameter method was used for the KASCADE-Grande standalone data analysis, where $k$ is basically the ratio of electron and muon numbers for the individual showers taking into account the incident zenith angle. The distribution of the $k$-parameter as a function of energies shows the evolution of the composition and it allows to separate the heavy and light primaries on an event-by-event. The energy and mass of the primary particle are calibrated with a set of simulations, which are obtained, separately, for each hadronic interaction model. Using the same hadronic interaction model, we define the separation $k$-parameter and interpret the full data set.

\section{Results and discussion}

A resulting energy spectrum is shown in Fig. 3 based on the interaction models of EPOSLHC [13] (dashed lines) and QGSJet-II-04 [14] (marks). By the combined analysis we obtain these spectra for an energy range of more than three orders of magnitude by means of one coherent analysis. The obtained spectra based on calibrations obtained using the QGSJet-II-04 confirm the 

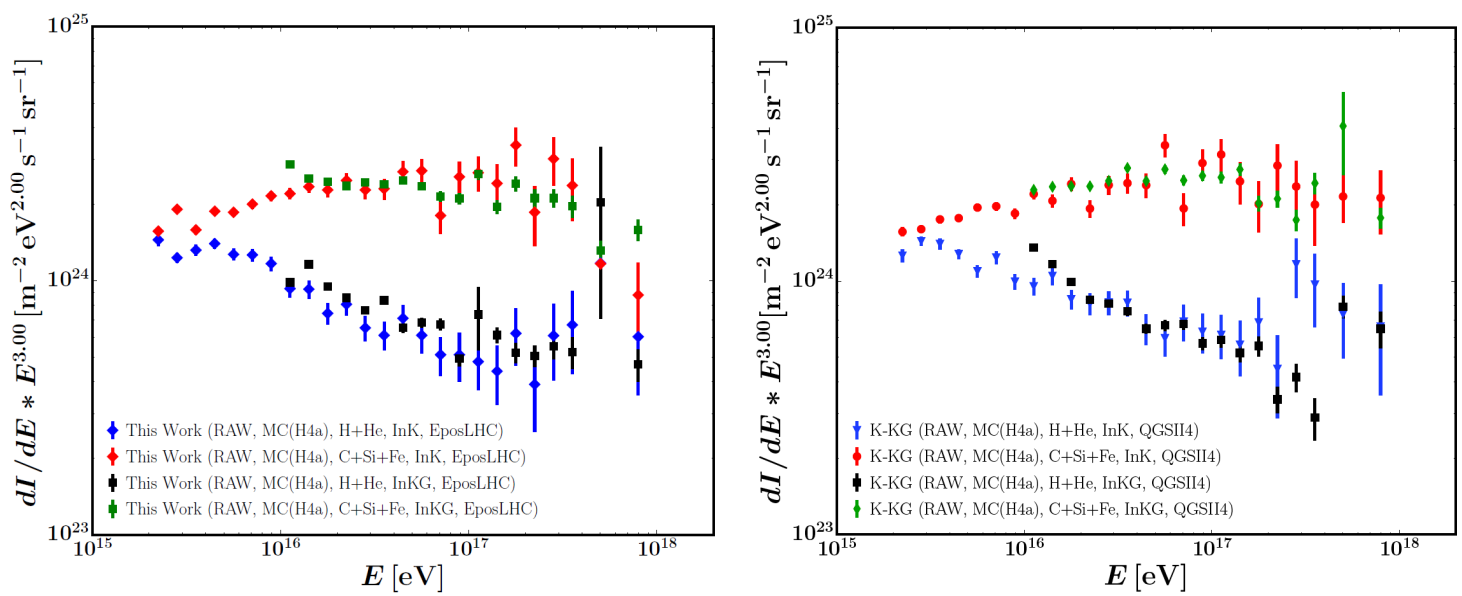

Figure 4: Reconstructed spectra for heavy and light components are shown for simulated data using EPOSLHC (left) and QGSJET-II-04 (right) as the hadronic interaction models and the H4a model [16] as the astrophysical model. The spectra have been obtained with the combined reconstruction, but by means of two data sets defined by the shower core being located in KASCADE and KASCADE-Grande, respectively.

earlier observation of all features: the light and heavy knees at around 3 and $100 \mathrm{PeV}$, and the lightankle at about $10 \mathrm{PeV}$ and $100 \mathrm{PeV}$, respectively. The results based on the EPOS-LHC confirm all these features as well. However, relative abundances of the light and heavy primary spectra for the post-LHC models show a significant difference between hadronic models.

It is worth mentioning that for the comparison of EPOS-LHC with QGSJet-II-04, the spectra of light components $(\mathrm{H}+\mathrm{He})$ agree well, however, not for the spectra of heavy primaries $(\mathrm{C}+\mathrm{Si}+\mathrm{Fe})$. It might be implied that the post-LHC models describe the proton-proton interaction better than the nucleus-nucleus interaction. Therefore, to build a consistent model, some additional theoretical prediction is required to be taken into account, in particular, in the nucleus-nucleus interaction.

Another possible reason for the discrepancy of the heavy spectra is that the muon component is not sufficiently well described [15].

\section{Hadronic interaction models}

We studied more about the source of the differences in interpreting the same data with different hadronic interaction models with following approaches: For each interaction model, separately, a data set was generated with the simulated spectra of the five different primaries $\mathrm{H}, \mathrm{He}, \mathrm{C}, \mathrm{Si}$, and $\mathrm{Fe}$ in an assumption corresponding to the predictions of an astrophysical model, which is the H4a model [16] in this case. I.e., the simulated data have been weighted, so that their true spectra match the predictions of the H4a model. In addition, for cross-checking the results, we divide two data sets based on the location of the shower core: One set containing events located within KASCADE (InK) and the other one with events located in KASCADE-Grande (InKG). In particular, the measurements of muons are different for these two data sets. The advantage for this is that in one of the samples we obtain the muon number by measuring muons close to the shower core (up to $200 \mathrm{~m}$ ) and for the other sample by measuring muons far $(300-700 \mathrm{~m}$ ) from 

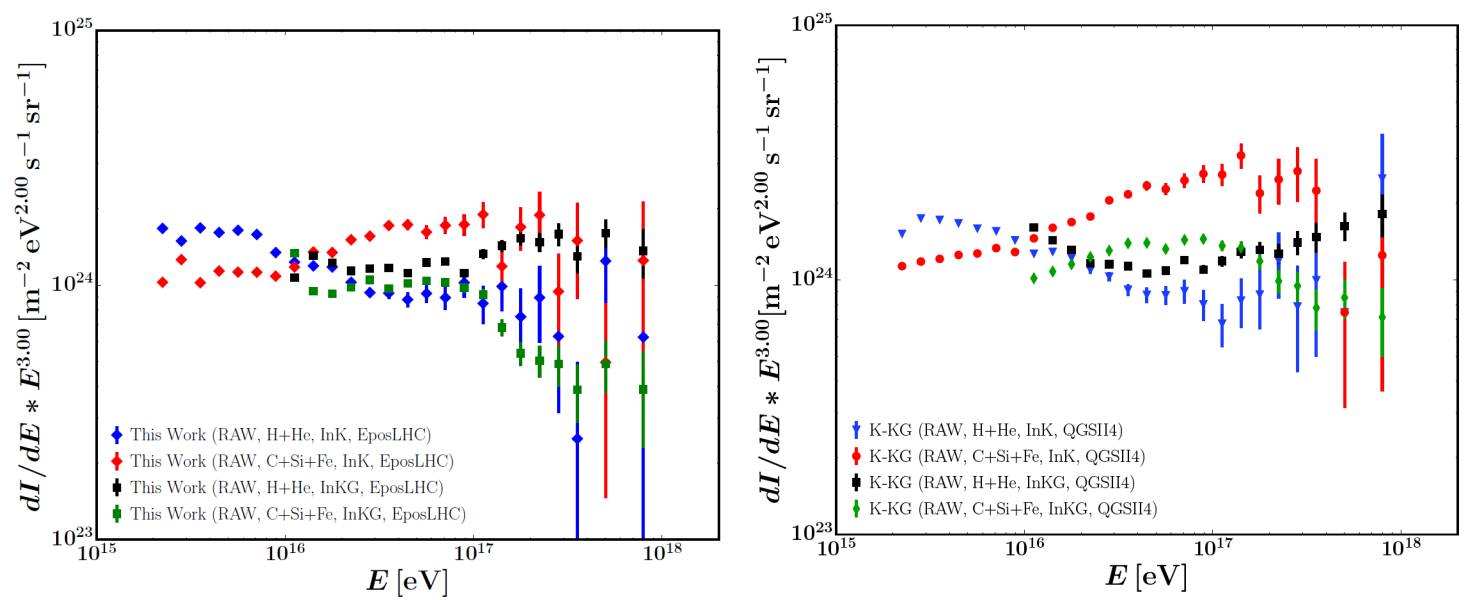

Figure 5: Reconstructed spectra for heavy and light components are shown for measured data using calibration based on EPOS-LHC (left) and QGSJET-II-04 (right) as the hadronic interaction models. The spectra have been obtained with the combined reconstruction, but by means of two data sets defined by the shower core being located in KASCADE and KASCADE-Grande, respectively.

the shower core. Other shower parameters are obtained with similar systematics in both samples. The purpose of this procedure is to see how the different hadronic interaction models QGSJet-II04 [14] and EPOS-LHC [13] compare to each other and to the data. We apply the full simulated data including the detector response to the above mentioned reconstruction. The results show an internal consistency of the models within systematic uncertainties for the two studied interaction models (see Fig. 4).

Applying the reconstruction to the two measured data samples, independently, the spectra show significant differences. Figure 5 shows the reconstructed spectra for heavy and light components using calibration based on EPOS-LHC (left) and QGSJET-II-04 (right) as the hadronic interaction models, where two data sets defined by the shower core being located in KASCADE and KASCADE-Grande were used, respectively. A systematic deficiency appears obviously for the model to describe the muon contents of the showers. Either there are too few muons predicted in the center of the extensive air showers or too few at larger distances. In other words, the lateral distribution of muons is predicted to be too flat. The presented investigation is neither sensitive to the total number of muons nor to give a statement of which of these two cases is more probable. However, these are strong hints that there are deficiencies in describing the slope of the lateral distribution of muons in air showers.

\section{Conclusion}

The combined analysis of the data from both KASCADE and KASCADE-Grande arrays significantly improved the reconstruction of the shower parameters and extends the fiducial area. We obtained a consistent and more detailed estimation of the energy spectrum and composition of cosmic rays in the energy range of $\mathrm{PeV}$ to $\mathrm{EeV}$. The spectral features observed beforehand, i.e. knees and ankles, of the light and heavy mass group spectra have been confirmed. 
In addition, an important investigation to interpret the combined data was performed with most recent hadronic interaction models of EPOS-LHC and QGSJET-II-04, which take into account the latest data of the Large Hadron Collider. These models have converged to a consistent description of the light mass group of cosmic ray primaries. However, their different extrapolation from protonproton interactions to proton-nucleus and nucleus-nucleus interactions still results in spectra of heavy primaries that differ from each other.

A possible reason for this discrepancy between measurement and simulation might lie in the lateral distribution of muons. The slope of the lateral density function used to estimate the total number of muons is a fixed parameter and has been derived from simulated data. It turns out that the predicted lateral distribution is too flat compared to measured data resulting in an overestimation of the number of muons for events located in KASCADE and an underestimation for events located in KASCADE-Grande.

\section{References}

[1] T. Antoni et al., KASCADE collaboration, Astropart. Phys. 24 (2005) 1-25

[2] W.D. Apel et al., KASCADE-Grande collaboration, Astrop. Phys. 36 (2012) 183

[3] W.D. Apel et al., KASCADE-Grande collaboration, Phys. Rev. Lett. 107 (2011) 171104

[4] W.D. Apel et al., KASCADE-Grande collaboration, Phys. Rev. D 87 (2013) 081101

[5] T. Antoni et al., KASCADE collaboration, Nucl. Instr. Meth. A513 (2003) 490

[6] G. Navarra et al., KASCADE-Grande collaboration, Nucl. Instr. Meth. A518 (2004) 207

[7] S. Schoo, Doctoral Dissertation, KIT Karlsruhe (2016); DOI:10.5445/IR/1000055797

[8] W.D. Apel et al., KASCADE-Grande collaboration, Nucl. Instr. and Meth. A620 (2010) 202

[9] D. Heck et al., Rep. FZKA 6019, Forschungszentrum Karlsruhe (1998)

[10] K. Greisen, Prog. Cosmic Ray Phys. III 1-141 (1956)

[11] A.A. Lagutin and R.I. Raikin, Nucl. Phys. Proc. Suppl. 97 (2001) 274

[12] S. Schoo et al, KASCADE-Grande collaboration, PoS(ICRC2015) 263

[13] T. Pierog, Iu. Karpenko, J. M. Katzy, E. Yatsenko, and K. Werner, Phys. Rev. C 92 (2015) 034906

[14] S. Ostapchenko, PRD 83 (2011) 014018; EPJ web. conf. 52 (2013) 02001

[15] J.C. Arteaga et al., KASCADE-Grande collaboration, these proceedings

[16] T.K. Gaisser, Astropart. Phys. 35 (2012) 801 